\title{
A Strategic Analysis of Global Warming: Theory and Some Numbers
}

\author{
Prajit K. Dutta* And Roy RadneR**
}

August 11, 2002. This Version: March 5, 2005****

\begin{abstract}
We model the global warming process as a dynamic commons game in which the players are countries, their actions at each date produce emissions of greenhouse gases, and the state variable is the current stock of greenhouse gases. The theoretical analysis is complemented by a calibration exercise. The first set of results establishes theoretically and then empirically the over-emissions due to a "tragedy of the commons." The power of simple sanctions to lower emissions and increase welfare is then examined as is the effect of cost asymmetry. Finally, a complete theoretical charactrization is provided for the best equilibrium and it is shown that it has a very simple structure; it involves a constant emission rate through time.

JEL Numbers - D900, Q540, C730

Keywords - Global Warming, Dynamic Games, Tragedy of the Common, Calibration

* Department of Economics, Columbia University, New York, NY 10027;

email: pkd1@columbia.edu (Author for correspondence purposes.)

**Stern School of Business, New York, University, New York, NY 10012;

email: rradner@stern.nyu.edu
\end{abstract}

\section{INTRODUCTION}

1.1. Background and Principal Issues. The gravest environmental challenge facing humanity today is global climate change. ${ }^{1}$ Its costs are potentially huge and its effect worldwide. ${ }^{2}$ Furthermore, there is time-pressure on the world's governments to deal with the problem now.

****This paper has benefitted from the comments of seminar audiences at the University of Michigan, University of Rochester, Boston University, CalTech, UCLA, Columbia University, The Stockholm School of Economics and New York University. Of course, all errors are our very own.

${ }^{1}$ Here are three facts about global climate change:

1. In the last 140 years, global surface temperatures have risen 0.6 degrees $\mathrm{C}$

2. All ten of the warmest recorded years have been since 1990, including each of the last seven years.

3. The Intergovernmental Panel on Climate Change (IPCC) predicts that - without significant changes - temperatures will rise between 1.5 and 5.5 degrees C by 2100 .

(see the BBC Science report, December 6, 2004, in http://news.bbc.co.uk/2/hi/science/nature/4061871.stm.)

${ }^{2}$ The importance of global warming is underlined in the discussions at the two worldwide summits - Rio de Janeiro in 1992 and Kyoto in 1997 - as well as in the follow-up sessions at the Hague in 2000 and in Bonn and Marrakesh in 2001 and Buenos Aires in 2004. 
This paper studies a simple strategic model of global climate change and asks: what are the economic consequences of "unregulated" climate change? Is there a simple set of self-enforcing guidelines that improve on the current situation? And by how much? What is the best "regulation" or treaty that governments can sign? What is the effect of the many cost differences across countries? Most of the analysis is theoretical but we also report results from a numerical calibration of the model.

Various factors contribute to global warming, but the major one is increase in greenhouse gases (GHGs) - primarily, carbon dioxide - so called because they are transparent to incoming shortwave solar radiation but trap outgoing longwave infrared radiation. Increased carbon emissions due to the burning of fossil fuel, in order to generate energy, is commonly cited as the principal immediate cause of global warming. ${ }^{3}$ One important fact to keep in mind here is that carbon dioxide buildup is very slow to reverse itself. ${ }^{4}$

Although there is considerable uncertainty about the exact costs of global warming, the two principal sources will be a rise in the sea-level and climate changes. The former may wash away low-lying coastal areas such as Bangladesh and the Netherlands. Climate changes are more difficult to predict; tropical countries will become more arid and less productive agriculturally; there will be an increased likelihood of hurricanes, fires and forest loss; and there will be the unpredictable consequences of damage to the natural habitat of many living organisms. Note that these costs are quite unevenly distributed; the "South" will suffer more than the "North" and even within a single country there will be disparities.

On the other hand, emission abatement imposes its own costs. Higher emissions are typically associated with greater GDP and consumer amenities (via increased energy usage). Reducing emissions will require many or all of the following costly activities: cutbacks in energy production, switches to alternative modes of production, investment in more energy efficient equipment, investment in $R \& D$ to generate alternative sources of energy, etc.

The principal features of the global climate change problem are:

- The Global Common - although the source of carbon buildup is localized, it is the total stock of GHGs in the global environment that will determine the amount of warming.

\footnotetext{
${ }^{3}$ The IPCC 2001 report declared unequivocally "most of the observed warming over the last fifty years is likely to have been due to the increase in greenhouse gas concentrations." Cumulative carbon emissions on account of fossil-fuel use rose from 0.1 gigatons to 5.5 gigatons between 1880 and 1980. In the same period, emissions from forest depletion rose from 0.75 to 2.5 gigatons. (For these and related figures, see Grubler and Fujii, 1991).

${ }^{4}$ In the article "In Kyoto the Subject is Climate, the Forecast is Storms", The New York Times, November 27, 1997, one climatologist described current worldwide efforts to contain global warming as trying to "turn an oil tanker in a sea of jello."
} 
- Irreversibility - since the stock of greenhouse gases depletes slowly, the effect of current emissions can be felt into the distant future.

- Asymmetry - some regions will suffer more than others.

- Nonlinearity - the costs can be very nonlinear; a rise in one degree may have little effect but a rise in several degrees may be catastrophic.

The theoretical framework that accommodates all of these features is an asymmetric dynamic commons model with the global stock of greenhouse gases as the (common) state variable. We study such a model with all the above characteristics except non-linearity and, additionally, two other features:

- A StrategicModel - Although the players (countries) are relatively numerous, there are some very large players, and blocks of like-minded countries, like the U.S., Western Europe, and Japan. That warrants a strategic analysis. Furthermore a lesson of history is that the only good international treaties are those that are incentive-compatible for the signatories. (Indeed an objection to the Kyoto Protocol - although not the one commonly cited - is that it is not incentive-compatible; more on this in Section 8. $)^{5}$

- A Simple Model - The model we study embeds a very simple linear description of how global climate change affects the physical and economic world. This is done for two reasons: one, for a theoretical exercise to have any chance of informing policy-makers in even one country, leave alone 200, its conclusions have to be simple. (Indeed all the proposals at Kyoto or follow-up conferences have involved simple across the board cuts in emissions.) Second, that also allows us to calibrate our model and derive some numerical guideposts.

The research reported in this paper is a first step of an ongoing program (see also the further discussion in Section 8 and especially, Dutta and Radner (2004a-d) as well as Dutta, Park and Radner (2004, in preparation).

1.2. The Results. In the model, each country adds to a global stock of greenhouse gases (GHGs) by its emission of those gases. The existing stock depreciates at a constant rate over time. Emissions arise from the production and consumption of energy, and other productive activities, and hence higher emissions are associated with, at least up to a point, higher benefits. The cost of global climate change is linear in the amount of GHGs in the earth's atmosphere. Both the benefits as well as

\footnotetext{
${ }^{5}$ All of the existing analyses of global climate change are either non-strategic or static. For reviews of the literature and further references, see Heal (1997), Finus (2001), and Barrett (2003).
} 
the costs vary across countries. The model has a small set of parameters that guide it, and drawing on estimates for those parameters that are available in the literature, we are also able to calibrate the model.

We start with two baseline results (Section 3). We first show that Global Pareto optimal (GPO) emission profiles involve a constant emission of GHGs by each country, i.e., constant across GHG levels, and hence through time. ${ }^{6}$ Call such a Pareto optimal emission vector $\widehat{a}$.

Next we characterize a Markov Perfect equilibrium (MPE) that is qualitatively similar to the GPO solution; it too involves a constant emission at rate $a^{*}$ by each country. Not surprisingly, this equilibrium, which we call Business as Usual (BAU), exhibits the tragedy of the commons; $a^{*}$ is strictly higher than the GPO vector $\widehat{a}$. The numerical estimates for this over-emission are illustrative. For base-line parameter values, the GPO is between 8 and $10 \%$ lower than the BAU level for developed economies and between 28 and 34\% lower for developing economies and China. (Contrast this with the Kyoto sanctioned 5\% cut for developed economies.) Corresponding welfare loss - in per period terms - ranges from $0.1 \%$ for base-line costs to $2 \%$ for higher cost estimates.

We then turn to the main question(s) of this paper: can countries do better than globally warm at the BAU rate, i.e., are there equilibria that are Pareto-improvements over the BAU solution? Put differently, can countries sign a Kyoto-like agreement that slows down the pace of global warming and is honored by all signatories?

In Section 4 we start with Nash or BAU reversion trigger strategy equilibria - since these are the simplest to implement. The two main theoretical results here show that for all discount factors and all countries, one can do strictly better; the highest welfare level is strictly higher than the BAU one and the minimum incentive-compatible emission levels are strictly lower than the BAU levels. Second, if discount factors are high enough, then, in fact, the GPO emission levels are themselves sustainable.

We then provide empirical evidence for these results. For the base-line parameter values, the minimum sustainable emissions are already lower than the GPO levels for all regions except Eastern Europe (and some FSU allies such as North Korea). In particular, the greatest discrepancies arise for Russia and Ukraine where the GPO emission levels are, respectively, 39\% and 40\% lower than the BAU emission levels but the maximum sustainable cuts from BAU levels are only $20 \%$ and $19 \%$ respectively.

The effect of asymmetry is studied in Section 5 by analyzing trigger strategy equilibria. Since there is no obvious measure of asymmetry with many countries, we restrict attention to two countries - or regions - and measure asymmetry by the

\footnotetext{
${ }^{6}$ The GPO emission is constant on account of the linearity in the cost function; that, coupled with the constant depreciation rate, implies that the marginal valuation of a unit of emission is independent of the existing stock of GHG.
} 
difference in (marginal) costs of GHG. Starting with the no-asymmetry case where the GPO itself is sustainable we show that increasing asymmetry leads to strict welfare loss, i.e., leads to a reduction in the total payoffs of the two countries. Again we provide numerical analogs of these results by focussing on China and the United States.

The last two sections are purely theoretical. In Section 6 we characterize the full set of subgame perfect equilibria (SPE). We show that the best equilibria have a remarkably simple structure - they too have a constant emission vector along the equilibrium path, say $\bar{a}$ (and this rate is strictly lower than the BAU rate $a^{*}$ ). The sanctions that support such a low emission level is the worst equilibrium for each player. That equilibrium is asymmetric (even in a symmetric game) and has an interesting two-stage structure. In the first stage, and for exactly one period, all countries other than the deviant country emit a "very high" amount, in excess of the BAU level. This behavior punishes all countries. For the sanctioning countries, the compensation is that in the second stage play proceeds to an equilibrium they prefer (and one that the sanctioned country does not). This is the equilibrium that maximizes a weighted sum of (equilibrium) payoffs, with zero weight on the sanctioned country. In such an equilibrium, the country with zero weight typically has to settle for very low emissions. This then is the long-term cost that a country suffers by breaking an agreement; its emission "quota" is permanently reduced.

Finally in Section 7 we consider emission strategies that are Markovian but not necessarily constant and we demonstrate the existence of MPE that have simultaneously "basins of tragic attraction" - levels of GHGs that are inoptimally high which once the system gets into there are no incentives to get out of - as well as "basins of low GHG buildup" - regions of carbon buildup that are no more than what a global planner would recommend. In other words, we show that there are MPE that have simultaneously characteristics of "bad" and "good" equilibria; where the system ends up depends then on where it started.

Section 8 concludes with some suggestions for extending the model to make it more realistic, including the incorporation of technological change, economic and population growth, and a literature review. Proofs are gathered in the Appendix Section 9.

\section{A Simple Model}

In this section we present a simplified model to illustrate the basic strategic ideas. In this model there is no population growth and no possibility of changing the emissions producing technologies in each country. ${ }^{7}$ However, the countries may differ in their "sizes," their emissions technologies, and their preferences.

\footnotetext{
${ }^{7}$ Population growth is studied in Dutta and Radner (2004b) while certain kinds of technological changes are allowed in Dutta and Radner (2004a).
} 
There are $I$ countries. The emission of (a scalar index of) greenhouse gases during period $t$ by country $i$ is denoted by $a_{i}(t)$. [Time is discrete, with $t=0,1,2, \ldots$, ad inf.] Let $A(t)$ denote the global (total) emission during period $t$;

$$
A(t)=\sum_{i=1}^{I} a_{i}(t) .
$$

The total (global) stock of greenhouse gases (GHGs) at the beginning of period $t$ is denoted by $g(t)$. The law of motion for the total GHG is

$$
g(t+1)=A(t)+\sigma g(t)
$$

where $\sigma$ is a given parameter $(0<\sigma<1)$. We may interpret $(1-\sigma)$ as the fraction of the beginning-of-period stock of GHG that is dissipated from the atmosphere during the period. The "surviving" stock, $\sigma g(t)$, is augmented by the quantity of global emissions, $A(t)$, during the same period.

Suppose that the utility of country $i$ in period $t$ is

$$
v_{i}(t)=h_{i}\left[a_{i}(t)\right]-c_{i} g(t)
$$

The function $h_{i}$ represents, for example, what country $i$ 's gross national product would be at different levels of its own emissions, holding the global level of GHG constant. This function reflects the costs and benefits of producing and using energy as well as the costs and benefits of other activities that have an impact on the emissions of GHGs, e.g., the extent of forestation. For a given population there will be an optimal level of energy use, forestation, etc., and hence an optimal level of emissions. It therefore seems natural to assume that $h_{i}$ is a strictly concave $C^{2}$ function that reaches a maximum and then decreases thereafter. ${ }^{8}$

The parameter $c_{i}>0$ represents the marginal cost to the country of increasing the global stock of GHG. Of course, it is not the stock of GHG itself that is costly, but the associated climatic conditions. In a more general model, the cost would be nonlinear. The total payoff (utility) for country $i$ is

$$
v_{i}=\sum_{t=0}^{\infty} \delta^{t} v_{i}(t) .
$$

For the sake of simplicity, we have taken the discount factor, $\delta$, to be the same for all countries. At time $t$, a history of play is an enumeration of past GHG levels up to

\footnotetext{
${ }^{8}$ However, none of the results depend on $h$ having a finite argmax.
} 
and including the current one as well as past emissions for all countries. ${ }^{9}$ A strategy for country $i$ at time $t$, call it $\sigma_{i t}$, is a measurable map from the set of histories to the set of emissions. A strategy for the whole game, denoted by $\sigma_{i}$, is a sequence of such strategies: $\sigma_{i} \equiv\left[\sigma_{i 0}, \sigma_{i 1}, \ldots \sigma_{i t}, \ldots\right] .{ }^{10}$ Let $\sigma$ denote a profile of strategies, one for each country: $\sigma=\left(\sigma_{1}, \sigma_{2}, \ldots \sigma_{I}\right)$. A particularly simple strategy for country $i$ is to condition her action at period $t$ only on the state at that period; such a strategy is called a Markovian strategy.

Each profile $\sigma$, and the initial level of $\mathrm{GHG}, g_{0}$, determine in an obvious manner country $i^{\prime} s$ payoff in period $t$; denote this payoff by $v_{i}\left(t ; \sigma, g_{0}\right)$. Thus associated with each profile is a total discounted payoff for each player

$$
v_{i}\left(\sigma, g_{0}\right) \equiv \sum_{t=0}^{\infty} \delta^{t} v_{i}\left(t ; \sigma, g_{0}\right) \text {. }
$$

As usual, a profile of strategies $\sigma^{*}$ forms a Nash Equilibrium (or just an equilibrium) from the initial GHG level $g_{0}$ if no player can increase her total discounted expected payoff by unilaterally changing her strategy. A profile $\sigma^{*}$ forms a subgame perfect Equilibrium (SPE) if after every history the continuation strategies of $\sigma^{*}$ form a Nash equilibrium. If a profile of Markovian strategies forms a Nash equilibrium from every initial state then it is called a Markov Perfect Equilibrium (MPE); note that an MPE is also an SPE.

2.1. An Illustrative Example. The theoretical results of this paper will be illustrated throughout by use of a calibrated model using parameter values that have been considered in the literature. The aim is to provide thereby some numerical orders of magnitude for the results stated here. In this subsection we will briefly describe the numerical analysis and in subsequent sections we will present the numerical analogs immediately following the appropriate theoretical results.

The numerical example is taken from a much more extensive analysis by Sangwon Park (Park 2004). In his model, 184 countries are grouped for calibration purposes into eight regions: the United States, Western Europe, Other High Income, Eastern Europe, Middle Income, Lower Middle Income, China, and Lower Income. [This grouping enables Park to utilize the data and estimates provided by Nordhaus and Boyer (2000).] The base year is $1998(t=0)$. Referring to Eq. 3, the utility

\footnotetext{
${ }^{9}$ If there is imperfect monitoring, then player $i^{\prime} s$ history will only contain her own past actions. In this paper we shall restrict attention to the perfect monitoring case, although our results on Markovian strategies will apply to the case of imperfect monitoring, as well. This is because, by definition, Markovian strategies only condition on the observable part of history, i.e., the total stock of GHGs.

${ }^{10}$ For expositional ease we restrict attention to pure strategies. None of the results would change by allowing mixed strategies.
} 
of country $i$ net of warming costs, its gross national product after subtracting the amount of capital needed to maintain the capital stock at its 1998 level, $h_{i}$, is given by:

$$
h_{i}\left[a_{i}(t)\right]=\phi_{i} K_{i}^{\gamma_{i}} L_{i}^{1-\gamma_{i}-\beta_{i}} \frac{a_{i}(t)^{\beta_{i}}}{f_{i}}-p_{i} \frac{a_{i}(t)}{f_{i}}
$$

where $K_{i}$ and $L_{i}$ are the capital and labor inputs, respectively; $f_{i}$ is the emission factor of country $i$ and $p_{i}$ is the price of "energy". To explain the last two terms in greater detail, imagine that energy is a proxy for a scalar index of emissionsproducing inputs, measured in coal-equivalent metric tons. Then for every unit of energy there are associated $f_{i}$ units of emissions. Countries differ in their emission factors - countries with cleaner technologies have lower $f_{i}$ than those with "dirtier" technologies. The Greek letters are parameters. These parameters as well as the price of energy, $p_{i}$, is the same for all countries in the same region (that is what defines a region) while the damage coefficient $c_{i}$ is based on a combination of country and group-specific data and is drawn from Fankhauser (1995). All three production inputs - capital, labor, and energy - are country-specific.

All countries have the same discount factor, $\delta$, which for sensitivity analysis has been varied between 0.97 and 0.995 . These would seem to bracket the values commonly discussed in the literature, motivated by different interpretations of $\delta$ as reflecting "social values" or the returns on investment. In what follows utility is measured in 1990 U.S. dollars and emissions are measured in gigatons of carbon.

\section{TWO BENCHMARKS}

In this section we characterize two benchmarks - the global Pareto optimuma, and a simple Markov Perfect Equilibrium, called "Business As Usual".

3.1. Global Pareto Optima. Let $x=\left(x_{i}\right)$ be a vector of positive numbers, one for each country. A Global Pareto Optimum (GPO) corresponding to $x$ is a profile of strategies that maximizes the weighted sum of country payoffs,

$$
v=\sum_{i} x_{i} v_{i}
$$

which we shall call global welfare. Without loss of generality, we may take the weights, $x_{i}$, to sum to 1 .

Theorem 1. Let $\hat{V}(g)$ be the maximum attainable global welfare starting with an 
initial GHG stock equal to $g$; then

$$
\begin{aligned}
\hat{V}(g) & =\widehat{u}-w g, \\
w & =\frac{1}{1-\delta \sigma} \sum_{i} x_{i} c_{i}, \\
\widehat{u} & =\frac{\sum_{i} x_{i} h_{i}\left(\hat{a}_{i}\right)-\delta w \hat{A}}{1-\delta}, \\
\hat{A} & =\sum_{i} \hat{a}_{i},
\end{aligned}
$$

where $\hat{a}_{i}$ is determined by

$$
x_{i} h_{i}^{\prime}\left(\hat{a}_{i}\right)=\delta w .
$$

(It is assumed that this last equation has a solution.) Furthermore, country $i$ 's GPO strategy is to use a constant emission equal to $\hat{a}_{i}$ in all periods and after all histories.

Theorem 1 states that, independently of the level of GHG, $g$, each country should emit an amount $\hat{a}_{i}$. The fact that the optimal emission is constant follows from the linearity of the model in $g$. Notice that on account of the linearity in the gas buildup equation - Eq. 2 - a unit of emission in period $t$ can be analyzed in isolation as a surviving unit of size $\sigma$ in period $t+1, \sigma^{2}$ in period $t+2, \sigma^{3}$ in period $t+2$, and so on. On account of the linearity in cost, these surviving units add $\left(\sum_{i} x_{i} c_{i}\right) \times \delta \sigma$ in period $t+1,\left(\sum_{i} x_{i} c_{i}\right) \times(\delta \sigma)^{2}$ in period $t+2$, and so on, i.e., the marginal lifetime cost is $\frac{1}{1-\delta \sigma} \sum_{i} x_{i} c_{i}$ or $w$. And that marginal cost is independent of $g$.

3.2. A Markov-Perfect Equilibrium: "Business as Usual".

This MPE shares the feature that the equilibrium emission rate of each country is constant in time, and it is the unique MPE with this property. We shall call it the "Business-asUsual" equilibrium.

Theorem 2. ("Business-as-Usual Equilibrium). Let $g$ be the initial stock of GHG. For each country $i$, let $a_{i}{ }^{*}$ be determined by

$$
\begin{aligned}
h_{i}^{\prime}\left(a_{i}{ }^{*}\right) & =\delta w_{i}, \\
w_{i} & =\frac{c_{i}}{1-\delta \sigma},
\end{aligned}
$$

and let its strategy be to use a constant emission equal to $a_{i}{ }^{*}$ in each period; then this strategy profile is a MPE, and country $i$ 's corresponding payoff is

$$
\begin{aligned}
V_{i}^{*}(g) & =u_{i}^{*}-w_{i} g, \\
u_{i}^{*} & =\frac{h_{i}\left(a_{i}^{*}\right)-\delta w_{i} A^{*}}{1-\delta}, \\
A^{*} & =\sum_{j} a_{j}{ }^{*}
\end{aligned}
$$


The intuition for the existence of an MPE with constant emissions is similar to that for the GPO solution. As long as other countries do not make their emissions contingent on the level of GHGs, country $i$ has a constant marginal lifetime cost to emissions. There are, however, other MPEs in which countries do condition on $g$, and some of them will be described in Section 7.

3.3. Comparison of the GPO and Business as Usual. The preceding results enable us to compare the emissions in the GPO with those in the Business-as-Usual MPE:

$$
\begin{array}{rlrl}
\text { GPO }: & & h_{i}^{\prime}\left(\hat{a}_{i}\right)=\frac{\delta \sum_{j} x_{j} c_{j}}{x_{i}(1-\delta \sigma)}, \\
\text { BAU }: & h_{i}^{\prime}\left(a_{i}{ }^{*}\right)=\frac{\delta c_{i}}{1-\delta \sigma} .
\end{array}
$$

Since

$$
x_{i} c_{i}<\sum_{j} x_{j} c_{j}
$$

it follows that

$$
\frac{\delta c_{i}}{1-\delta \sigma}<\frac{\delta \sum_{j} x_{j} c_{j}}{x_{i}(1-\delta \sigma)} .
$$

Since $h_{i}$ is concave, it follows that

$$
a_{i}^{*}>\hat{a}_{i}
$$

Note that this inequality holds except in the trivial case in which all welfare weights are zero (except one). In other words, there is a tragedy of the commons whenever there is some externality to emissions. In turn, all this follows from Eq. 8, which says that in the BAU equilibrium each country only considers its own marginal cost and ignores the cost imposed on other countries on account of its emissions; in the GPO solution that additional cost is, of course, accounted for. It follows that the GPO is strictly Pareto superior to the MPE for an open set of welfare weights $x_{i}$ (and leads to a strictly lower steady-state GHG level for all welfare weights).

3.4. Numerical Results on the GPO and BAU. In this subsection we present numerical results on the emission levels and welfare values - especially in a comparative sense - of the GPO and BAU solutions. As indicated above, the results are derived by using numerical estimates for parameter values that have been used elsewhere by Fankhauser (1995) and Nordhaus and Boyer (2000). We start with the benchmark case in which $\delta=0.97$ and cost coefficients $c_{i}$ are as given in Fankhauser. This case has been calibrated so that the BAU matches available data and estimates for 1998. The benchmark GPO then uses the same parameter values and 
initial conditions. (Throughout, in computing GPO trajectories, we have taken the country-welfare weights $x_{i}$ to be equal to 1 . The consequences of using other welfare weights have been explored in Park (2004).)

Table 1 - Benchmark Case $\left(\delta=0.97\right.$, cost $=$ Fankhauser $c_{i}$, Year $\left.=1998\right)$

$\begin{array}{cccc}\text { Region/Emissions } & B A U(G t c) & \text { GPO }(G t c) & \% \text { Difference }\left(\frac{B A U-G P O}{B A U}\right) \\ \text { United States } & 1.50 & 1.36 & 9 \% \\ \text { Western Europe } & 0.86 & 0.79 & 8 \% \\ \text { Other High Income } & 0.59 & 0.53 & 9 \% \\ \text { Eastern Europe } & 0.74 & 0.45 & 39 \% \\ \text { Middle Income }(M I) & 0.41 & 0.36 & 12 \% \\ \text { Lower MI } & 0.58 & 0.41 & 30 \% \\ \text { China } & 0.85 & 0.56 & 34 \% \\ \text { Lower Income } & 0.66 & 0.48 & 28 \% \\ \text { Total } & 6.18 & 4.93 & 20 \%\end{array}$

The most striking results are: a) BAU emission levels are most significantly higher (relative to GPO) for Eastern Europe, presumably on account of Soviet-era energy technologies; b) the next significant departures are for China and Lower Middle Income countries (that include India) and this is particularly worrisome if the major growth spurt of the next fifty years comes from those countries, which will then move them way past Eastern Europe in over-emission levels; and c) that the United States and Western Europe account for over $40 \%$ of current total emissions and hence a cut-back to GPO levels in those regions alone can significantly narrow the overall GPO-BAU gap.

The differences between GPO and BAU are magnified if either of the two basic parameters are increased. Consider an increase in the discount factor to 0.995 (reflecting the idea that environmental discount factors ought to be closer to 1 for inter-generational equity reasons); this is Table 2. The main findings while qualitatively similar - Eastern Europe is the greatest problem - show that China and Lower Income Countries have almost as much over-emissions. This could pose a huge problem down the line if these economies continue to grow strongly. ${ }^{11}$

\footnotetext{
${ }^{11} \mathrm{~A}$ change in the second parameter, raising the cost estimates to $5 \mathrm{x}$ Fankhauser - reflecting possible catastrophic costs - increases the range of over-emissions to $56 \%$ (Western Europe) to $90 \%$ (Eastern Europe).
} 
A Strategic Analysis of Global Warming: Theory and Some Numbers

Table 2 - Sensitivity Analysis I $\left(\delta=0.995\right.$, cost $=$ Fankahauser $c_{i}$, Year $=$ 1998)

$\begin{array}{cccc}\text { Region/Emissions } & \text { BAU }(G t c) & \text { GPO }(G t c) & \% \text { Difference }\left(\frac{B A U-G P O}{B A U}\right) \\ \text { United States } & 1.50 & 1.15 & 23 \% \\ \text { Western Europe } & 0.86 & 0.69 & 20 \% \\ \text { Other High Income } & 0.59 & 0.46 & 22 \% \\ \text { Eastern Europe } & 0.74 & 0.27 & 64 \% \\ \text { Middle Income }(M I) & 0.41 & 0.29 & 28 \% \\ \text { Lower MI } & 0.58 & 0.27 & 54 \% \\ \text { China } & 0.85 & 0.32 & 62 \% \\ \text { Lower Income } & 0.66 & 0.32 & 52 \% \\ \text { Total } & 6.18 & 3.76 & 39 \%\end{array}$

We now turn to value comparisons, i.e., to the question: how much is the welfare loss due to over-emission and its consequent increase in global temperatures? Unlike Tables 1 and 2 which report a one-shot picture, the welfare analysis takes account of the infinite future. In particular, what is computed is the present discounted average value of GNP less carbon costs - i.e., $(1-\delta) V_{i}$ - so that when $\delta$ is close to 1 the discounted average value is close to the long-term yearly average. Note that if $2 \%$ of a country's GNP were invested every year, the rate of growth of its economy would be dramatically increased.

Table 3 provides value loss estimates for $\delta=0.97$ while Table 4 provides the parallel estimates for $\delta=0.995$. The carbon cost parameters are drawn from Fankhauser (1997) - denoted FC - and the sensitivity analysis is to vary them between his estimates $(=\mathrm{FC})$ and five times his estimates $(=5 \mathrm{XFC})$ :

Table 3 - Value Loss when $\delta=0.97$

$\begin{array}{cccccc}\text { Value Loss/Cost Parameter } & F C & 2 X F C & 3 X F C & 4 X F C & 5 X F C \\ (G P O-B A U) / B A U \text { in } 1998 \text { dollars } & 0.11 \% & 0.4 \% & 0.82 \% & 1.34 \% & 1.95 \%\end{array}$

Table 4 - Value Loss when $\delta=0.995$

Value Loss/Cost Parameter $\quad F C \quad 2 X F C \quad 3 X F C \quad 4 X F C \quad 5 X F C$

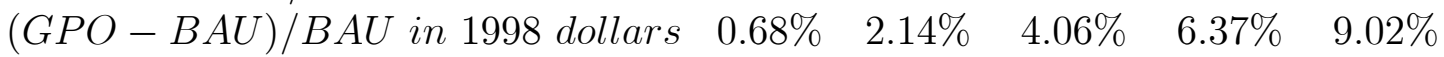

\section{Other Equibria I - BAU Sanctions}

In this section we take a first step towards characterizing other equilibria in our model; we analyze the third-best problem: maximize a weighted sum of country payoffs when the emission policies are incentive-compatible under the threat of BAU 
emissions. There are three main results. First, for all discount factors, the thirdbest solution qualitatively mirrors the BAU and GPO solutions; there is a constant emission level $a_{i}^{\prime}$ that country $i$ emits, independently of the stock of GHGs. Second, the set of sustainable emissions includes the BAU level but is strictly larger than that. In particular, the minimum incentive-compatible emission level is strictly lower than the BAU level, and that is true for every country. Hence, BAU sanctions are strictly welfare improving for all countries and all discount factors. Third, if discount factors are high enough, then, in fact, the GPO emission levels are themselves the third-best solution. Finally, we provide empirical evidence for these results.

4.1. BAU Sanctions: Third-Best and Minimum Emissions. Let $x=\left(x_{i}\right)$ be a vector of positive numbers, one for each country. A Third-Best Optimum (TBO) corresponding to $x$ is a profile of "norm" strategies that maximizes the weighted sum of country payoffs,

$$
v=\sum_{i} x_{i} v_{i}
$$

subject to BAU reversion, i.e., subject to the constraint - detailed below - that should any country $i$ not follow the norm, all countries would switch to BAU emissions forever after from the following period. As before, and without loss of generality, we may take the weights, $x_{i}$, to sum to 1 . The following result characterizes the TBO:

Proposition 1 There exists a vector of constant emission levels a' such that country i's TBO strategy is to use a constant emission equal to $a_{i}^{\prime}$ in all periods and after all histories in which no country defects, where $a_{i}^{\prime}$ belongs to a set that satisfies the incentive constraint

$$
h_{i}\left(\widetilde{a}_{i}\right)-\delta w_{i}\left(\widetilde{a}_{i}+\delta \sum_{j \neq i} \widetilde{a}_{j}\right) \geq h_{i}\left(a_{i}^{*}\right)-\delta w_{i}\left(a_{i}^{*}+\delta \sum_{j \neq i} a_{j}^{*}\right), \forall i
$$

It might help to sketch the derivation of the simple incentive constraint above. Consider any constant emission level $\widetilde{a}=\widetilde{a}_{1}, \widetilde{a}_{2}, \ldots \widetilde{a}_{I}$. It can be easily shown that this emission level is sustainable as an equilibrium norm provided

$h_{i}\left(\widetilde{a}_{i}\right)-c_{i} g+\delta\left[\widetilde{u}_{i}-w_{i}\left(\sigma g+\sum_{j} \widetilde{a}_{j}\right)\right] \geq h_{i}\left(a_{i}\right)-c_{i} g+\delta\left[u_{i}^{*}-w_{i}\left(\sigma g+a_{i}+\sum_{j \neq i} \widetilde{a}_{j}\right)\right], \forall a_{i}, i$

where $\widetilde{u}_{i}$ and $u_{i}^{*}$ are the present discounted values of emission policies that emit at constant rates of, respectively, $\widetilde{a}$ and $a^{*}$ into the infinite future - recall that in the event of a deviation, the threat is to revert to BAU emissions $a^{*}$ forever. In turn, the above equation can be simplified to

$$
h_{i}\left(\widetilde{a}_{i}\right)-\delta w_{i} \widetilde{a}_{i}+\delta \widetilde{u}_{i} \geq h_{i}\left(a_{i}\right)-\delta w_{i} a_{i}+\delta u_{i}^{*}, \forall a_{i}, i
$$


From Eq. 8 - the characterization of the BAU emission level - it follows that the deviation profits - the RHS of the equation above - is maximized at $a_{i}^{*}$. Using that fact and the definitions of $\widetilde{u}_{i}$ and $u_{i}^{*},{ }^{12}$ the incentive constraint can be rewritten in the form above.

It is immediate that the BAU emission policy is sustainable by the threat of BAU reversion - of course! - since the inequality is trivially satisfied when $\widetilde{a}_{i}=a_{i}^{*}$. What is also not very difficult to show is that the GPO emission policy also becomes sustainable at a high enough $\delta$. Formally, we have:

Proposition 2 a) The highest welfare that is achievable under the threat of $B A U$ emissions is at least as high as $u^{*}$.

b) Suppose that the GPO values under equal country weighting, $\left(x_{i}=x_{j}\right.$ for all i,j) Pareto-dominates the BAU solution for all high $\delta \geq \delta^{\prime}$ Then, there is a cut-off discount factor $\widetilde{\delta} \in\left(\delta^{\prime}, 1\right)$ such that, above it, the GPO emission policy is sustainable as an equibrium norm.

Consider the minimum emission problem:

$$
\operatorname{Min} \sum_{i} \widetilde{a}_{i}
$$

over all incentive-compatible emission levels, i.e., over all $\widetilde{a}$ that satisfy the incentive constraint above. The next proposition shows that there is a unique solution to the problem and, for all $\delta$, it is strictly smaller than the BAU emission level $a^{*}$.

Proposition 3 a) There is a unique emission level a that is the minimum emission level sustainable by the threat of BAU reversion.

b) For all $\delta$, the minimum emission level is strictly lower than the BAU level, i.e., $\underline{a}<<a^{*}$.

4.2. BAU Sanctions: Numerical Evidence on Minimum Emissions. In this subsection we present empirical evidence on the emission cuts that are sustainable under the threat of BAU sanctions. We focus on minimum emission levels $\underline{a}$ and show that this is for most regions even lower than the GPO levels. There is one significant exception to this and that is Eastern Europe where GPO levels are higher than the lowest that BAU sanctions will deliver. We also present come country-specific evidence. Again countries from the ex-Soviet Bloc are the ones where significant cuts appear most difficult to implement; for Russia and Ukraine the GPO emission levels are, respectively, $39 \%$ and $40 \%$ lower than the BAU emission levels but the maximum sustainable cuts are only $20 \%$ and $19 \%$ respectively.

In Tables 5 and 6 below, MIN refers to the minimum emission level $\underline{a}$.

\footnotetext{
${ }^{12}$ Recall - see Eq. $9-u_{i}^{*}=\frac{h_{i}\left(a_{i} *\right)-\delta w_{i}}{1-\delta} a_{j}^{*}$ and $\widetilde{u}_{i}=\frac{h_{i}\left(\widetilde{a}_{i}\right)-\delta w_{i} \sum \widetilde{a}_{j}}{1-\delta}$.
} 
Table 5 - Minimum Emissions Under BAU Sanctions; $\delta=0.97$ and Fankhauser costs:

$\begin{array}{cccc}\text { Region/Emissions } & \text { BAU (Gtc) } & \text { GPO (Gtc) } & \text { MIN (Gtc) } \\ \text { United States } & 1.50 & 1.36 & 1.22 \\ \text { Western Europe } & 0.86 & 0.79 & 0.67 \\ \text { Other High Income } & 0.59 & 0.53 & 0.45 \\ \text { Eastern Europe } & 0.74 & 0.45 & 0.54 \\ \text { Middle Income }(M I) & 0.41 & 0.36 & 0.28 \\ \text { Lower MI } & 0.58 & 0.41 & 0.32 \\ \text { China } & 0.85 & 0.56 & 0.45 \\ \text { Lower Income } & 0.66 & 0.48 & 0.43 \\ \text { Total } & 6.18 & 4.93 & 4.36\end{array}$

Table 6 - A Comparison of Minimum Emissions and GPO Emissions for Selected Countries:

$\begin{array}{cc}\text { Country/Emissions } & \frac{M I N-G P O}{B A U}(\%) \\ \text { UnitedStates } & -9 \% \\ \text { Brazil } & -31 \% \\ \text { China } & -21 \% \\ \text { India } & -9 \% \\ \text { Korea DPR } & +22 \% \\ \text { Poland } & +5 \% \\ \text { Russia } & +17 \% \\ \text { Ukraine } & +21 \%\end{array}$

\section{ASYMMETRY}

One critical feature of the climate change problem is that countries differ greatly in the consequences they are likely to suffer. Whereas low-lying places like Bangladesh may see parts of their countries washed away by rising sea-waters, or equatorial countries may become uninhabitably hot, northern regions of the world such as parts of North America and northern Europe will bear limited costs and may even benefit from longer growing seasons and lower heating costs in the winter. In this section we analyze the question: (how) does asymmetry (in costs) limit the extent of sustainable cuts? Optimal cuts? What are the welfare consequences of asymmetry?

As in the last section, we will limit attention to equilibria that are sustainable by the threat of reversion to the BAU equilibrium. For this section, we will focus on

- Two Countries, i.e., $I=2$

The restriction is imposed for clarity. We study two countries - or two regions because it is easier to define asymmetry in terms of a single-dimensional parameter 
(namely the difference in the $\operatorname{costs} c_{1}$ and $c_{2}$ ) and it is less obvious what we mean by an "increase" in asymmetry when we have $I$ costs.

5.1. Incentives and Welfare. No matter what emissions policy we consider, when country $i^{\prime} s$ marginal cost to global warming, $c_{i}$ increases, its total and marginal lifetime cost increases. As we saw in Section 3, the emission in any period $t$ can be treated in isolation a and $\operatorname{costs} \frac{c_{i}}{1-\delta \sigma}$. Hence when $c_{i}$ increases, everything else being equal, country $i$ should be willing to cut emissions since benefits are unchanged and the marginal cost is higher. And conversely for a decrease in costs.

The problem though is that everything else does not actually remain unchanged when costs change. In particular, the sanction, i.e., the BAU solution, itself changes with the cost. In principle then, the comparative statics of incentives are unclear; whereas, a higher cost makes cheating on a treaty less profitable it also makes the sanction to be suffered less severe. In this subsection we show that nevertheless the first effect always triumphs; a higher cost country will agree to every cut that a lower cost country agrees to - and possibly more.

Without loss of generality, let us consider cost changes in which it is $c_{1}$ that decreases while $c_{2}$ increases. It might help to think of country 1 as a proxy for the developed North and country 2 as the developing South.

Consider any constant emissions policy with emissions at rate $\widetilde{a}$. As we saw in the previous section, this policy is incentive-compatible - under BAU reversion - if

$$
h_{i}\left(\widetilde{a}_{i}\right)-\delta w_{i}\left(\widetilde{a}_{i}+\delta \widetilde{a}_{j}\right) \geq h_{i}\left(a_{i}^{*}\right)-\delta w_{i}\left(a_{i}^{*}+\delta a_{j}^{*}\right), i=1,2, i \neq j
$$

The policy is strictly incentive-compatible for country $i$ if the inequality holds strictly. A study of the incentive constraint yields the following conclusion: the country whose cost increases is willing to make larger cuts and the exact opposite is true for countries whose cost decreases.

Theorem 3. For every emission vector $\widetilde{a}$ less than the BAU vector $a^{*}$, a decrease in $c_{1}$ and a simultaneous increase in $c_{2}$ changes incentives as follows: if $\left(\widetilde{a}_{1}, \widetilde{a}_{2}\right)$ is incentive compatible (under $\operatorname{costs} c_{1}$ and $c_{2}$ ) then it becomes strictly incentive-compatible for country 2 (the "South") under the new costs. Likewise, if $\widetilde{a}$ is just compatible for country 1 (the "North") before the cost change, then it is no longer incentivecompatible under the new costs.

Recall the symmetric third-best problem:

$$
\operatorname{Max} V_{1}(g ; a)+V_{2}(g ; a),
$$

where $V_{i}(g ; a)$ is country $i^{\prime} s$ lifetime payoff from emission policy $a($.$) - and this emission$ policy is incentive compatible under the threat of BAU reversion. Denote cost 
asymmetry by a single-dimensional parameter $d$ where $d=0$ corresponds to equal costs and cost asymmetry increases as $d$ increases (the North's costs decrease and the South's increase).${ }^{13}$. The third-best welfare result is:

Theorem 4. Suppose that the GPO is sustainable as a third-best equilibrium when costs are equal, $d=0$. Overall welfare, as measured by the sum of country payoffs are decreasing in cost asymmetry. It remains unchanged for small asymmetry - till a cut-off asymmetry level $\widehat{d}$ - and thereafter strictly decreases.

5.2. Asymmetry: Some Numbers. In this subsection the two countries are the US (country 1) and China (country 2) whose actual costs are quite similar to each other. A table illustrates the third-best as it changes with asymmetry $d$.

Table 7 - Third-Best Emission Levels as a function of cost difference $d$ :

$\begin{array}{ccc}d & U S(G t C) & \text { China }(G t C) \\ 0.2 & 1.46400 & 0.77206 \\ 0.3 & 1.46369 & 0.77201 \\ 0.4 & 1.46369 & 0.77201 \\ 0.5 & 1.46048 & 0.77971 \\ 0.6 & 1.45601 & 0.79646 \\ 0.7 & 1.45332 & 0.81295\end{array}$

6. Other Equilibria II - A Complete Characterization of SPE

In this section we will characterize all possible subgame perfect equilibria (SPE) of the model. We will do that in two steps. In Section 6.1 we will characterize the SPE payoff correspondence. This characterization will be then used in Section 6.2 to study the extreme equilibria - the best and the worst equilibria. Although the theoretical analysis is complete, numerical estimates of extreme equilibria - and especially the worst equilibrium - are more difficult to compute. That exercise is left for future research.

6.1. The Equilibrium Payoff Correspondence. We will show that the SPE payoff correspondence has a surprising simplicity; the set of equilibrium payoffs at a level $g$ is a simple linear translate of the set of equilibrium payoffs from some benchmark level, say, $g=0$. Consequently, it will be seen that the set of emission levels that can arise in equilibrium from level $g$ is identical to those that can arise from equilibrium play at a GHG level of 0 . Note the fact that the set of equilibrium

\footnotetext{
${ }^{13}$ Furthermore, the parametrization is done in such a fashion that the total cost, $w_{1}+w_{2}$, remains unchanged.
} 
possibilities is invariant to the level of $g$, is perfectly consistent with the possibility that in a particular equilibrium, emission levels vary with $g$. (Indeed we will see some of those equilibria in Section 7.) However, the invariance property will make for a particularly simple characterization of the best and worst equilibria.

Let $\Xi(g)$ denote the set of equilibrium payoff vectors with initial state $g$, i.e., each element of $\Xi(g)$ is the payoff to some SPE starting from $g$.

Theorem 5. The equilibrium payoff correspondence $\Xi$ is linear; there is a compact set $U \subset \Re^{I}$ such that for every initial state $g$

$$
\Xi(g)=U-\left\{w_{1} g, w_{2} g, \ldots w_{I} g\right\},
$$

where $w_{i}=\frac{c_{i}}{1-\sigma \delta}, i=1, . . I$. In particular, consider any SPE, any period $t$ and any history of play up until $t$. Then the payoff vector for the continuation strategies must necessarily be of the form

$$
V-\left(w_{1} g_{t}, w_{2} g_{t}, \ldots w_{I} g_{t}\right)
$$

where $V \in U$ (and $g_{t}$ is the state at period $t$ ).

The theorem is proved by way of a bootstrap argument. We presume that a (candidate) payoff set has this invariance and show that the linear structure of the model confirms the conjecture. Consequently, we generate another candidate payoff set - which is also state invariant. Then we look for a fixed point of that operator. ${ }^{14}$

6.2. Extreme Equilibria. We will now use the result of the previous subsection to characterize the best - and the worst - equilibria in the global climate change game. Consider the second-best problem (from initial state $g$ and for a given vector of welfare weights $\left.x=\left(x_{i} ; i=1, . . I\right)\right)$, i.e., the problem of maximizing a weighted sum of equilibrium payoffs:

$$
\max \sum_{i=1}^{I} x_{i} V_{i}(g), V(g) \in \Xi(g) .
$$

Note that we consider all possible equilibria, i.e., we consider equilibria that choose to condition on current and past GHG levels as well as equilibria that do not. The result states that the best equilibrium need not condition on GHG levels:

\footnotetext{
${ }^{14}$ In other words, we employ a generalized version of the Abreu, Pearce, and Stachetti (1990) operator to generate the SPE correspondence. We need to generalize the APS argument since that was formulated for repeated games alone.
} 
Theorem 6. There exists a constant emission level $\bar{a} \equiv \bar{a}_{1}, \bar{a}_{2}, \ldots . \bar{a}_{I}$ - such that no matter what the initial level of GHG, the second-best policy is to emit at the constant rate $\bar{a}$. In the event of a deviation from this constant emissions policy by country $i$, play proceeds to $i^{\prime} s$ worst equilibrium. Furthermore, the second-best emission rate is always strictly lower than the BAU rate, i.e., $\bar{a}<a^{*}$. Above a critical discount factor (less than 1), the second-best rate coincides with the GPO emission rate $\widehat{a}$.

The theorem is attractive for two reasons: first, it says that the best possible equilibrium behavior is no more complicated than BAU behavior; so there is no argument for delaying a treaty (to cut emissions) merely because the status quo is simple. Second, the cut required to implement the second-best policy is an across the board cut - independently of anything else, country $i$ should cut its emissions by the amount $a_{i}^{*}-\bar{a}_{i}{ }^{15}$

Sanctions will be required if countries break with the second-best policy ${ }^{16}$ and without loss of generality we can restrict attention to the worst such sanction. We turn now to a characterization of this worst equilibrium (for, say, country $i$ ). One definition will be useful for this purpose:

Definition 1. An $i$-less second-best equilibrium is the solution to a second-best problem in which the welfare weight of $i$ is set equal to zero, i.e., $x_{i}=0$.

By the previous theorem, every such problem has a solution in which on the equilibrium path, emissions are a constant. Denote that emission level $a\left(x_{-i}\right)$ :

Theorem 7. There exists a "high" emission level $\overline{\bar{a}}(i)$ (with $\sum_{j \neq i} \overline{\bar{a}}_{j}(i)>\sum_{j \neq i} a_{j}^{*}$ ) and an $i$-less second-best equilibrium $a\left(x_{-i}\right)$ such that country $i$ 's worst equilibrium is:

1. Each country emits at rate $\overline{\bar{a}}_{j}(i)$ for one period (no matter what $g$ is), $j=1, . . I$.

2. From the second period onwards, each country emits at the constant rate $a_{j}\left(x_{-i}\right), j=1, . . I$.

And if any country $k$ deviates at either stages 1 or 2 , play switches to $k^{\prime} s$ worst equilibrium from the very next period after the deviation.

\footnotetext{
${ }^{15}$ Our model operates at the aggregative level alone and, in particular, we do not address the issue of how national governments will implement cuts that they agree to (in the national interest). However, it seems quite likely that an across the board cut will be easier to implement - and will be perceived to be fairer to all - than one which is sensitively tied to levels of GHG.

${ }^{16} \mathrm{~A}$ major criticism of the Kyoto accord is that it did not incorporate sanctions and hence would never be carried out. For details, see Barrett (2003, Chapter 15) and the further discussion in Section 8.
} 
Put another way, for every country $i$, a sanction is made up of two emission rates, $\overline{\bar{a}}(i)$ and $a\left(x_{-i}\right)$. The former imposes immediate costs on country $i$. The way it does so is by increasing the emission levels of countries $j \neq i$. The effect of this is a temporary increase in incremental GHG but due to the irreversibility of gas accumulation, a permament increase in country $i^{\prime} s$ costs, enough of an increase to wipe out any immediate gains that the country might have got from the deviation. Of course this additional emission also increases country $j^{\prime} s$ costs. For the punishing countries, however, this increase is offset by the subsequent permanent change, the switch to the emission vector $a\left(x_{-i}\right)$, which permamently increases their quota at the expense of country $i^{\prime} s$.

The fact that there is a temporary loosening of environmental regulations as part of environmental sanctions is reminiscent of GATT rules where tariffs can be temporarily imposed by countries that seek to punish illegitimate trade practices on the part of others.

\section{Greenhouse Trap}

In every equilibrium that we have studied so far - BAU, the third and second-best each country emits at a constant rate regardless of GHG level. Hence, the dynamics of every such equilibrium is also simple; at a constant cumulative rate $A$, the stock of greenhouse gases (GHGs) converges to a steady-state of $\frac{A}{1-\sigma}$. Put yet another way, the current GHG level has no long-term implication.

In this section we demonstrate the richness of the model by identifying some MPE in which current GHG levels matter, MPE in which there is a so-called "greenhouse trap". If the world starts below some critical level of GHG, say $\widetilde{g}$, then it grows no farther than that level. However if the system starts above $\widetilde{g}$ - or somehow crosses into the higher region - then greenhouse gases are trapped into growing - and eventually grow to the BAU steady-state.

The key to these equilibria is a richer interaction between the (Markov) emission levels of country $i$ and the rest of the world. In particular, we will consider emission policies that are Markovian but not constant, say an emission policy (vector) such as $a(g)$. Now country $i$ has an incentive - everything else being equal - to emit in such a fashion that the global stock of GHG grows towards a region where $a_{-i}($.$) are$ lower. Of course every country has such an incentive and so each country will wish to drive $g_{t}$ towards a region where emission levels are low for the other countries. Hence, the conjecture for the group as a whole is that there will be MPE with regions of "abnormally" low emissions and every country will a) have an incentive to stay in such regions once the system gets there and b) have an incentive to participate in pushing the system towards such regions.

The conjecture is almost correct. We will show that there are indeed such equilibria - in fact there are many - each of which has such a "good" region of low emissions. 
Furthermore, from most - but not all - initial GHG levels outside this good region, countries will have an incentive to drive the system to the good region. The one additional complication is that in order for there to be a good region there must also be some "bad" region (of high emissions) from which countries do not find their way to the good region. The presence of a bad region - and the associated spectre of landing there - is what keeps the countries honest in the good region. ${ }^{17}$

To keep the exposition simple, we are going to only present results for the symmetric case. ${ }^{18}$ Accordingly, when we speak of the Pareto optimal solution we will refer to the symmetric solution - and to avoid clutter we will simply denote that solution by $\widehat{a}$ (with associated steady state $\widehat{g}$ ). We shall present two results of increasing generality.

Consider the following symmetric Markovian strategy $a($.$) : if the GHG level is$ below the Pareto optimal steady state $\widehat{g}$, emissions take the game immediately to that state. On the other hand, if the GHG level exceeds $\widehat{g}$, then emissions are at the (high) BAU level of $a^{*}$. In other words,

$$
\begin{aligned}
a(g) & =\frac{\widehat{g}-\sigma g}{I}, g \leq \widehat{g} \\
& =a^{*}, g>\widehat{g}
\end{aligned}
$$

In the terminology of the immediately preceding discussion, the region below the Pareto optimal steady state $\widehat{g}$ is the "good" region of (relatively) low emission levels whereas the region above is the "bad" region of high emissions. Consider the following condition ("L" for "large")), which says that the BAU emission level is sufficiently larger than the GPO level:

\section{Condition L:}

$$
\frac{a^{*}}{\widehat{a}}>\max \left(\frac{I}{I-1}, \frac{1}{1-\sigma}\right),
$$

where (recall) $I$ is the number of players and $\sigma$ is the persistence of $\mathrm{CO}_{2}$ in the earth's atmosphere. ${ }^{19}$

\footnotetext{
${ }^{17}$ Readers familiar with repeated games will note the obvious connection with the idea of historydependent punishments. The point to note though is that these are not history-dependent equilibria since they only depend on the GHG level and not on past emissions. (Hence they are more difficult to construct.) They are also more sparing in terms of informational requirements since they do not require $i$ to condition on (or even know) past emission levels of the other countries.

${ }^{18}$ The first of the two results is easily generalized to the asymmetric case.

${ }^{19}$ Since $\frac{I}{I-1}$ approaches 1 for $I$ large, Condition $L$ really boils down to $\frac{a^{*}}{\widehat{a}} \geq \frac{1}{1-\sigma}$ whenever we have a large number of players. This condition will always hold under standard asymptotic conditions on $h$. To see this note that simple algebra shows that $\frac{h^{\prime}(\widehat{a})}{h^{\prime}\left(a^{*}\right)}=I$ and hence $\frac{a^{*}}{\widehat{a}}$ is large whenever $I$ is large.
} 
Theorem 8. Suppose that Condition $L$ holds. Then there is a cut-off value of the discount factor - say $\widehat{\delta}$ - such that $a($.$) is an MPE for all \delta \geq \widehat{\delta}$. In such an equilibrium, the GHG level converges in one period to the Pareto optimal steady state $\widehat{g}$ if the initial level is below $\widehat{g}$ whereas it converges asymptotically to the BAU steady state $g^{*}$ if the initial level is above $\widehat{g}$.

The reader might wonder how useful this last theorem is if we suspect that the world is currently already past the Pareto optimal steady state. ${ }^{20}$ This leads to the question: are there MPE that have steady states less than the BAU steady state of $g^{*}$ (but higher than the desired - but unattainable - steady state of $\widehat{g}$ )?

We will now demonstrate the existence of MPE that differs in three ways from the one above. First, in addition to the good and bad regions, there will be a "latent good" region - a region of low GHG levels from which the stock will gradually grow till it reaches the good region (whereupon it will stay there). Second, the good region (with emissions lower than the BAU level $a^{*}$ ) will extend beyond the Pareto optimal steady state $\widehat{g}$; in fact it will extend quite close to the BAU steady state $g^{*}$. (So no matter where the world is today, however close to the worst possibility, we can still put the brakes on in an incentive-compatible way!) Third, we will considerably weaken Condition $L$.

Let $\widetilde{g}$ be any GHG level that is higher than $\widehat{g}$ but no more than $\frac{(I-1)}{I} g^{*} ; \widetilde{g}$ will be our candidate low steady state. We will consider Markov strategies in which stocks from a left neighborhood of $\widetilde{g}$ (to be defined shortly) come in one step to $\widetilde{g}$ and stocks above $\widetilde{g}$ exhibit BAU behavior:

$$
\begin{aligned}
\widetilde{a}(g) & =a^{*}, g>\widetilde{g} \\
& =\frac{\widetilde{g}-\sigma g}{I}, \widetilde{g}_{1} \leq g \leq \widetilde{g}
\end{aligned}
$$

where

$$
\widetilde{g}_{1} \equiv \frac{I-1}{I} \widetilde{g}
$$

For stocks lower than $\widetilde{g}_{1}$, the "latent good" region, the emission levels will be such that GHG levels grow (gradually) till they get into the $\left[\widetilde{g}_{1}, \widetilde{g}\right]$ region. These emission levels cannot however be solved for in closed form. Instead we will employ a fixed point argument to show the following:

Theorem 9. Suppose that

$$
\frac{a^{*}}{\widehat{a}}>\frac{I}{I-1}
$$

\footnotetext{
${ }^{20}$ It is unclear whether the world is past $\widehat{g}$ or not. Some of the public policy concern is not so much with current levels of GHG as with what level might eventually be attained at the current rates of accumulation.
} 
Then there is a cut-off value of the discount factor - say $\widetilde{\delta}$ - such that for all $\delta \geq \widetilde{\delta}$, there is a MPE $\widetilde{a}($.$) whose behavior above \widetilde{g}_{1}$ is as given above. Below $\widetilde{g}_{1}$, the stock grows although it remains below $\widetilde{g}$, i.e.,

$$
\sigma g+I \widetilde{a}(g) \in(g, \widetilde{g}), \text { for all } g<\widetilde{g}
$$

In such an equilibrium, the GHG level converges to the steady state $\widetilde{g}$ if the initial level is below it whereas it converges asymptotically to the BAU steady state $g^{*}$ if the initial level is above $\widetilde{g}$.

\section{Literature Review and Concluding Remarks}

Symmetric dynamic commons games have been studied by a number of authors, including Levhari and Mirman (1980), Sundaram (1989), Sobel (1990), Benhabib and Radner (1992), Rustichini (1992), Dutta and Sundaram (1992, 1993), Sorger (1998), etc. ${ }^{21}$ Although these papers have greatly added to our understanding of the problem, none of them offer a full characterization of equilibria. We do just that in this paper (and, additionally, analyze the effect of asymmetry).

A large volume of literature exists on the economics of climate change. A central question there is to determine the level of emissions that is globally optimal. An excellent example of this is Nordhaus and Boyer (2000). A smaller volume of literature emphasizes the need for treaties to be self-enforcing, i.e., the need for a strategic analysis of the problem. (But see Barrett (2003) and Finus (2001).) Where we depart from the existing strategic literature is in the dynamic modelling; we allow GHGs to accumulate and stay in the environment for a (possibly long) period of time. (Technically, existing analyses are all static one-shot games or purely repeated games which implies that the state variable, gas stock, remains constant over time.)

In terms of conclusions, our findings are similar in terms of welfare loss to other authors, including those who focus on the first-best issue. Nordhaus and Boyer (2000) calculate a United States loss from global climate change of the order of $0.5 \%$ of GDP (for doubling of pre-industrial levels); other estimates have been in the $1 \%$ to $2 \%$ range. This agrees with our findings of a decrease in global welfare (respectively, US welfare) in the range of $0.6 \%$ to $2 \%$ (respectively, $0.3 \%$ to $1 \%$ ) for different discount rates with our benchmark damage cost coefficients. Where we differ is in the size of the emission cuts that our model predicts. Whereas Kyoto had called for cuts in the $10 \%$ range relative to 1990 levels and some of the literature has proposed that even that is too much to be globally optimal - see (Nordhaus and Boyer) - our numerical computations ask for much deeper emission cuts. We conjecture that the dynamic element of our model - that gases can persist in the atmosphere for a hundred years

\footnotetext{
${ }^{21}$ Some of these papers allow asymmetry; however, none of them analyzes the effect of asymmetry on the equilibria.
} 
or more, which is in line with the known evidence - make the deeper cuts optimal. Furthermore, our results for both potential welfare increases and emission cuts are quite sensitive to increases in the damage cost coefficients.

The Kyoto Protocol appears not to be the basis of a self-enforcing treaty, principally because it does not build in any effective sanctions that would be applied if countries fail to meet their targets. This contrasts with GATT and its attendant institutional structure, the WTO. All that is said in the Kyoto protocol - in Article 18 - is that procedures and mechanisms for compliance should be determined by the parties at their first meeting and should include "an indicative list of consequences". Subsequently, at the Hague November 2000 meeting, the most popular proposal (which came from the Dutch Environment Minister Jan Pronk) was that countries would face an escalating series of target reductions in the future if they failed to comply in the current stage. A watered-down version of this proposal was adopted in Bonn in March 2001, after the United States had pulled out of the treaty. Yet even this version had several problems - in principle, countries could postpone retribution indefinitely, and the base from which the enhanced reduction would be required would be worked out in the future, etc. (See Finus (2001), Chapter 15.) One contribution of this paper is that it shows that fairly simple sanctions - BAU reversion - can be quite effective and even the most severe sanctions - the worst equilibria - are fairly simple.

In Dutta and Radner (2004b) we generalize our current model to allow for population change and demonstrate qualitatively similar theoretical results. In Dutta and Radner (2004a) we allowed for simple technological change and present some theoretical and numerical results on the GPO and BAU solutions. In Dutta and Radner (2004c) we incorporate capital accumulation (and technical change). The main question that we hope to address in that model is: (when) does the prevention of global warming slow down the rate of economic growth? A second question that we hope to look at is: (how) does asymmetry in the current level of economic development affect sustainability of agreements about emission cuts. We have some preliminary results on the first question but not a complete solution.

\section{APPENDIX - PROOFS}

\subsection{Global Pareto Optima and BAU Equilibrium. Proof of Theorem}

1: We shall show by dynamic programming arguments that the Pareto-optimal value function is of the form $\widehat{V}=\sum_{i=1}^{I} x_{i}\left[\widehat{u}_{i}-w_{i} \dot{g}\right]$. We need to be able to find the constants $\widehat{u}_{i}$ to satisfy:

$$
\sum_{i=1}^{I} x_{i}\left[\widehat{u}_{i}-w_{i} g\right]=\operatorname{Max}_{a_{1}, . . a_{I}} \sum_{i=1}^{I} x_{i}\left[h_{i}\left(a_{i}\right)-c_{i} g+\delta\left(\widehat{u}_{i}-w_{i}\left(\sigma g+\sum_{j=1}^{I} a_{j}\right)\right)\right]
$$


Collecting terms that need maximization we can reduce the equation above to

$$
\sum_{i=1}^{I} x_{i} \widehat{u}_{i}=M a x_{a_{1}, . . a_{I}} \sum_{i=1}^{I} x_{i}\left[h_{i}\left(a_{i}\right)-\delta w_{i} \sum_{j=1}^{I} a_{j}\right]
$$

It is clear that the solution to this system is the same for all $g$; call this (first-best) solution $\widehat{a}_{i}$. Elementary algebra reveals that

$$
\widehat{u}_{i}=\frac{h_{i}\left(\widehat{a}_{i}\right)-\delta w_{i} \sum_{j=1}^{I} \widehat{a}_{j}}{1-\delta} \text { and } w_{i}=\frac{c_{i}}{1-\delta \sigma}
$$

It is also obvious that $x_{i} h_{i}^{\prime}\left(\hat{a}_{i}\right)=\delta w$, where $w=\sum_{i=1}^{I} x_{i} w_{i}$.

Proof of Theorem 2: Consider any constant emission strategy for players other than $i$; call these constants $\bar{a}_{j}$. We shall now show by dynamic programming arguments that the best response value function is of the form $\bar{u}_{i}-w_{i} \dot{g}$, where $\bar{u}_{i}=\frac{h_{i}\left(a_{i}^{*}\right)-\delta w_{i}\left(a_{i}^{*}+\sum_{j \neq i} \bar{a}_{j}\right)}{1-\delta}$. We therefore need to show that:

$$
\bar{u}_{i}-w_{i} \dot{g}=\operatorname{Max}_{a_{i}}\left[h_{i}\left(a_{i}\right)-c_{i} g+\delta\left(\bar{u}_{i}-w_{i}\left(\sigma g+a_{i}+\sum_{j \neq i} \bar{a}_{j}\right)\right]\right.
$$

Collecting the terms that need maximization we can reduce the above equation to

$$
\bar{u}_{i}=\operatorname{Max}_{a_{i}}\left[h\left(a_{i}\right)-\delta w_{i} a_{i}\right]
$$

It is clear that the solution to this system is the same for all $g$ and equals $a_{i}^{*}$ where $h_{i}^{\prime}\left(a_{i}^{*}\right)=\delta w_{i}$.

9.2. Equilibria Sustained by BAU Sanctions. Proof of Proposition 1: Recall that in the third-best problem we maximize a weighted sum of the countries' payoffs

$$
v=\sum_{i} x_{i} v_{i}
$$

subject to BAU reversion. The proof follows the lines of that for the analogous result on the second-best, i.e., along the lines of the proof of Theorems 6 and 7 (see below). Evidently, equilibria that are sustained by the threat of BAU reversion are SPE and hence their values belong to the SPE payoff correspondence that we identified in Theorem $6-U-\left\{w_{1} g, w_{2} g, \ldots w_{I} g\right\}$ where $U$ is a compact subset of $\Re^{I}$. Define therefore the set of incentive-compatible emission vectors $a$ - associated with the threat of BAU reversion - as:

$$
A=\left\{a \in \Re_{+}^{I}: h_{i}\left(a_{i}\right)+\delta\left(u_{i}^{\prime}-w_{i} a_{i}\right) \geq h\left(\widetilde{a}_{i}\right)+\delta\left(u_{i}^{*}-w_{i} \widetilde{a}_{i}\right), \forall \widetilde{a}_{i}, i, \text { for some } u^{\prime} \in U\right\}
$$


where $u_{i}^{*}=\frac{h_{i}\left(a_{i}^{*}\right)-\delta w_{i} \sum_{j=1}^{I} a_{j}^{*}}{1-\delta}$ is the BAU equilibrium payoff.

The third-best equilibrium is therefore found by solving the following problem:

$$
\operatorname{Max}_{a_{i}, u_{i}} \sum_{i=1}^{I} x_{i}\left[h_{i}\left(a_{i}\right)+\delta\left[u_{i}^{\prime}-w_{i} \sum_{k=1}^{I} a_{k}\right]\right]
$$

subject to the incentive compatibility constraint Eq. 20. Since the constraint is independent of $g$ - and so is the objective function - it follows that the solution is as well. The proposition is proved.

Proof of Proposition 2: The only part left to prove is part b). Note that as $\delta \uparrow 1$, the incentive constraint, Eq. 20, becomes

$$
h_{i}\left(\widetilde{a}_{i}\right)-w_{i}(1)\left(\widetilde{a}_{i}+\sum_{j \neq i} \widetilde{a}_{j}\right) \geq h_{i}\left(a_{i}^{*}\right)-w_{i}(1)\left(a_{i}^{*}+\sum_{j \neq i} a_{j}^{*}\right), \forall i
$$

where $w_{i}(1)=\lim _{\delta \uparrow 1} w_{i}(\delta)=\lim _{\delta \uparrow 1} \frac{c_{i}}{1-\sigma \delta}=\frac{c_{i}}{1-\sigma}$. The expression on the LHS, $h_{i}\left(\widetilde{a}_{i}\right)-w_{i}(1) \sum_{j} \widetilde{a}_{j}$, is precisely the long-run average (LRA) value from a policy that emits at the constant rate $\widetilde{a}$. In particular then, if we pick the candidate $\widetilde{a}$ to be the GPO emission under the LRA criterion with equal weights for all countries, the incentive constraint Eq. 21 is satisfied by the definition of GPO, at $\delta=1$. Add to that a) that the LRA GPO is the limit of discounted average GPO values (see, Dutta (1991)) and b) the assumption that the equal weight GPO solution Pareto-dominates the BAU value for all high discount factors. The proof is complete.

Proof of Proposition 3: We shall first prove part b). Start then at the BAU solution $a^{*}$ and consider a reduction of every country's emission by an amount $\varepsilon$. We shall show that the emission vector $a^{*}-\varepsilon$ is sustainable by the BAU sanction for appropriately small but positive $\varepsilon$. Note that the LHS of the incentive constraint, Eq. 20, becomes

$$
h_{i}\left(a_{i}^{*}-\varepsilon\right)-\delta w_{i}\left(a_{i}^{*}-\varepsilon+\delta \sum_{j \neq i}\left(a_{j}^{*}-\varepsilon\right)\right)
$$

and that can be rewritten as

$$
h_{i}\left(a_{i}^{*}\right)-\delta w_{i}\left(a_{i}^{*}+\delta \sum_{j \neq i}\left(a_{j}^{*}-\varepsilon\right)\right)-\left[h_{i}^{\prime}\left(z_{i}^{*}\right)-\delta w_{i}\right] \varepsilon
$$

for some $z_{i}^{*} \in\left(a_{i}^{*}-\varepsilon, a_{i}^{*}\right)$. That in turn equals

$$
h_{i}\left(a_{i}^{*}\right)-\delta w_{i}\left(a_{i}^{*}+\delta \sum_{j \neq i} a_{j}^{*}\right)+\left\{\delta^{2} w_{i}(I-1)-\left[h_{i}^{\prime}\left(z_{i}^{*}\right)-\delta w_{i}\right]\right\} \varepsilon
$$


As $\varepsilon \downarrow 0, z_{i}^{*} \rightarrow a_{i}^{*}$. Since $h_{i}^{\prime}\left(a_{i}^{*}\right)-\delta w_{i}=0$, it follows that $\delta^{2} w_{i}(I-1)-\left[h_{i}^{\prime}\left(z_{i}^{*}\right)-\right.$ $\left.\delta w_{i}\right]>0$ for small $\varepsilon$. In particular, the incentive constarint

$$
h_{i}\left(a_{i}^{*}-\varepsilon\right)-\delta w_{i}\left(a_{i}^{*}-\varepsilon+\delta \sum_{j \neq i}\left(a_{j}^{*}-\varepsilon\right)\right) \geq h_{i}\left(a_{i}^{*}\right)-\delta w_{i}\left(a_{i}^{*}+\delta \sum_{j \neq i} a_{j}^{*}\right), \forall i
$$

holds. The proof of part b) is complete.

At the minimum incentive-compatible emission level, every IC constraint must hold with equality. Consider instead the alternative case, i.e., suppose the constraint is a strict inequality for country $j$. In that case we can further reduce that country's emission without violating its constraint. However lowering $j^{\prime} s$ emission only helps satisfy every other country's constraint. Hence, we will have lowered total emissions without violating incentive constraints, which is a contradiction.

Rewriting the incentive constraints as equalities yields

$$
h_{i}\left(a_{i}\right)-\delta w_{i}\left(a_{i}+\delta \sum_{j \neq i} a_{j}\right)=\Psi_{i}, \forall i
$$

where $\Psi_{i}=h_{i}\left(a_{i}^{*}\right)-\delta w_{i}\left(a_{i}^{*}+\delta \sum_{j \neq i} a_{j}^{*}\right)$. Let us suppose that $\underline{a}$ is a solution to the emission minimization problem and let $\underline{A}=\sum_{i} \underline{a}_{i}$. Rewriting the immediately preceding equation we have

$$
h_{i}\left(\underline{a}_{i}\right)-(1-\delta) w_{i} \underline{a}_{i}=\Psi_{i}+\delta^{2} w_{i} \underline{A}
$$

Given the strict concavity of $h$, it is evident that $\underline{a}_{i}$ is uniquely defined by the above equation. The proof is complete.

9.3. Asymmetry. Proposition 1 above greatly simplifies the incentive analysis for the third-best problem since we can now restrict ourselves to constant emissions policies and the consequent incentive constraint:

$$
h_{i}\left(a_{i}\right)-\delta w_{i}\left(a_{i}+\delta a_{j}\right) \geq h_{i}\left(a_{i}^{*}\right)-\delta w_{i}\left(a_{i}^{*}+\delta a_{j}^{*}\right), i, \text { and for } j \neq i
$$

Proof of Theorem 3: Consider a decrease in $w_{1}$ (to $w_{1}^{\prime}$ ) and a simultaneous increase in $w_{2}$. Denote $S_{i}(a)$ as the incentive slack of country $i$, i.e.,

$$
S_{i}(a)=h_{i}\left(a_{i}\right)-\delta w_{i}\left(a_{i}+\delta a_{j}\right)-\left[h_{i}\left(a_{i}^{*}\right)-\delta w_{i}\left(a_{i}^{*}+\delta a_{j}^{*}\right)\right]
$$

Similarly, denote the incentive slack under the new (lower) costs $S_{i}^{\prime}$. Evidently, for country 1 , the difference in the two slacks, $S_{1}^{\prime}(a)-S_{1}(a)$ equals

$$
\begin{aligned}
& \left\{h_{1}\left(a_{1}\right)-\delta w_{i}^{\prime}\left(a_{1}+\delta a_{2}\right)-\left[h_{1}\left(a_{1}\right)-\delta w_{i}\left(a_{1}+\delta a_{2}\right)\right]\right\} \\
& -\left\{h_{1}\left(a_{1}^{*^{\prime}}\right)-\delta w_{i}^{\prime}\left(a_{1}^{*^{\prime}}+\delta a_{2}^{*^{\prime}}\right)-\left[h_{1}\left(a_{1}^{*}\right)-\delta w_{i}\left(a_{1}^{*}+\delta a_{2}^{*}\right)\right]\right\}
\end{aligned}
$$


where $a^{*^{\prime}}$ is the BAU emission level for costs $w^{\prime}$. Call the term in the first (curly) bracket, \{\}$_{1}$ - and the second one \{\}$_{2}$. Simple algebra yields

$$
\{\}_{1}=\delta\left(w_{1}-w_{1}^{\prime}\right)\left[a_{1}+\delta a_{2}\right] .
$$

Note that $h_{1}\left(a_{1}^{*^{\prime}}\right)-\delta w_{1}^{\prime}\left(a_{1}^{*^{\prime}}\right)>h_{1}\left(a_{1}^{*}\right)-\delta w_{1}^{\prime}\left(a_{1}^{*}\right)$ by the definition of $a_{1}^{*^{\prime}}$. Furthermore, $a_{2}^{*^{\prime}}<a_{2}^{*}$ since country 2 's costs have gone up. Combining those two facts we have that

$$
\begin{aligned}
\{\}_{2} & >h_{1}\left(a_{1}^{*}\right)-\delta w_{1}^{\prime}\left(a_{1}^{*}+\delta a_{2}^{*}\right)-\left[h_{1}\left(a_{1}^{*}\right)-\delta w_{1}\left(a_{1}^{*}+\delta a_{2}^{*}\right)\right. \\
& =\delta\left(w_{1}-w_{1}^{\prime}\right)\left[a_{1}^{*}+\delta a_{2}^{*}\right]
\end{aligned}
$$

Since $a_{1}^{*}+\delta a_{2}^{*} \geq a_{1}+\delta a_{2}$, it further follows \{\}$_{2}>\{\}_{1}$, i.e., $S_{1}^{\prime}(a)-S_{1}(a)=\{\}_{1}$ -\{\}$_{2}<0$.

To prove the result for country 2 , we mimic the above argument except for the fact that we use instead the observations that i) $h_{2}\left(a_{2}^{*}\right)-\delta w_{2}\left(a_{2}^{*}\right)>h_{2}\left(a_{2}^{*^{\prime}}\right)-\delta w_{2}\left(a_{2}^{*^{\prime}}\right)$ and ii) that $a_{1}^{*^{\prime}}>a_{1}^{*}$ since country 1 's costs have gone down. The theorem is proved.

Proof of Theorem 4: Consider starting from the symmetric situation where $c_{1}=c_{2}$ or, equivalently, the symmetric situation where the lifetime costs are equal, i.e., $w_{1}=\frac{c_{1}}{1-\delta \sigma}=\frac{c_{2}}{1-\delta \sigma}=w_{2}$. Now consider varying the costs in a manner that the costs of country 1 "South" are increased while those of country 2 "North" are decreased. Let is measure the asymmetry in costs by the difference $d=c_{1}-c_{2}$ or, equivalently, the difference in lifetime $\operatorname{costs} \frac{d}{1-\delta \sigma}=\theta=w_{1}-w_{2}$. In other words, we shall consider an increase in asymmetry as exemplified by an increase in $d$ (or $\theta$ ). Furthermore, so as to ensure that the only effect is that of an increase in asymmetry, we shall hold constant the sum of the two costs $w_{1}+w_{2}$.

By hypothesis, the GPO is third-best when $w_{1}=w_{2}$. Even when the cost difference becomes positive, the GPO continues to be incentive-compatible for both countries as long as $d$ is small. However, beyond a cut-off value, it is no longer incentive-compatible for the low cost country 2 "South" (see Lemma 1). At that point, in the third-best solution only one of the incentive constraints binds with a strict inequality. Furthermore, and again from Lemma 1 above, that has to be the incentive constraint for country 1 (the higher cost country).

So consider two different $\theta^{1}$ and $\theta^{2}$, say $\theta^{1}>\theta^{2}$ and let $a^{1}=\left(a_{1}^{1}, a_{2}^{1}\right)$ and $a^{2}$ $=\left(a_{1}^{2}, a_{2}^{2}\right)$ be the corresponding third-best emission vectors. Since the incentive constraint for country 1 "South" is slack, it follows that if $\theta^{1}$ is chosen sufficiently close to $\theta^{2}$, then $a_{1}^{1}$ is also incentive compatible at $\theta^{2}$. By Lemma 1 above, country 2's incentive region shrinks as its costs decrease, i.e., its emission under $\theta^{1}, a_{2}^{1}$, must also have been incentive compatible at $\theta^{2}$. Putting the two together, the emission vector $a^{1}$ is incentive compatible at $\theta^{2}$ but the choice made for that parameter is $a^{2}$. That implies that total welfare is higher at $a^{2}$ than at $a^{1}$ at the parameter $\theta^{2}$. But since 
the total welfare function is unchanged as the cost difference changes - since $w_{1}+w_{2}$ is constant - it follows that total welfare is lower at $\theta^{1}$ compared to $\theta^{2}$.

9.4. All Equilibria. Proof of Theorem 5: We shall employ the generalization of the Dynamic Programming Bellman equation that was introduced by AbreuPearce-Stachetti (1990) to study the set of SPE in repeated games. Let us start with the conjecture that the correspondence of SPE payoffs, $V$, has the structure claimed in the result, i.e., is of the form $U-\left\{w_{1} g, w_{2} g, \ldots w_{I} g\right\}$ where $U$ is a compact subset of $\Re^{I}$. Let $\underline{u}^{i}$ denote the vector in $U$ that gives country $i$ the worst payoff, i.e.,

$$
\underline{u}^{i}=\min _{u_{i}}\{u \in U\}
$$

and denote $i^{\prime} s$ payoff in that minimum as $\underline{u}_{i}^{i}$. Define the set of incentive-compatible emission vectors $a$ - associated with this candidate equilibrium payoff set - as:

$$
A=\left\{a \in \Re_{+}^{I}: h_{i}\left(a_{i}\right)+\delta\left(u_{i}^{\prime}-w_{i} a_{i}\right) \geq h\left(\widetilde{a}_{i}\right)+\delta\left(\underline{u}_{i}^{i}-w_{i} \widetilde{a}_{i}\right), \forall \widetilde{a}_{i}, i, \text { for some } u^{\prime} \in U\right\}
$$

Now define the APS operator $B V$ (that takes the correspondence $V$ into another correspondence) as follows:

$$
\begin{gathered}
B V=\left\{v(.): \exists \text { selections } a(.) \text { and } u(., .) \text { satisfying } a(g) \in A \text {, and } u\left(g^{\prime}, g\right) \in U, \forall g^{\prime}, g\right. \\
\left.v_{i}(g)=h_{i}\left(a_{i}\right)-c_{i} g+\delta\left(u_{i}\left(\sigma g+\sum_{j=1}^{I} a_{j}(g), g\right)-w_{i}\left(\sigma g+\sum_{j=1}^{I} a_{j}(g)\right)\right)\right\}
\end{gathered}
$$

Note that $v($.$) can be rewritten as$

$$
v_{i}(g)=\left[h_{i}\left(a_{i}\right)+\delta\left(u_{i}\left(\sigma g+\sum_{j=1}^{I} a_{j}(g), g\right)-w_{i} \sum_{j=1}^{I} a_{j}(g)\right)\right]-w_{i} g
$$

Note from the incentive set $A$ that exactly the same set of actions satisfy the incentive constraint at every value of $g$. By hypothesis the "continuation payoffs" $u\left(g^{\prime}, g\right) \in U$, a set that is independent of $g$ as well. It follows that $B V$ is a correspondence of the same form as $V$, i.e., $B V=U^{\prime}-\left\{w_{1} g, w_{2} g, \ldots w_{I} g\right\}$ for some $U^{\prime}$ that is a subset of $\Re^{I}$. Standard arguments - using the Maximum theorem - can be employed to show that $U^{\prime}$ must be a compact set as well.

Start then with a set $U_{0}$ (or correspondence $V_{0}$ ) that is compact and "large", i.e., one that contains all feasible payoffs in the game. Define $U_{1}$ via the APS operator as above - $U_{1}$ is compact as well. It must also be non-empty because it must contain the BAU payoff $u^{*}$. By definition, $U_{1} \subset U_{0}$. Define $U_{n}$ recursively in this fashion. It is easy to check that the operator is monotone and hence $U_{n}$ is a decreasing sequence 
of non-empty compact sets. Associated with each is the correspondence $V_{n}=U_{n}$ $\left\{w_{1} g, w_{2} g, \ldots w_{I} g\right\}$. Let $U$ be the intersection of these $U_{n}$ sets. APS type arguments then show that $B V=V$ and that $V$ contains all SPE payoffs. The theorem is proved.

Proof of Theorem 6: Given the previous theorem, the second-best equilibrium is found by solving the following problem:

$$
\operatorname{Max}_{a, u(., g) \in U} \sum_{i=1}^{I} x_{i}\left[h_{i}\left(a_{i}\right)+\delta\left[u_{i}\left(\sigma g+\sum_{k=1}^{I} a_{k}, g\right)-w_{i} \sum_{k=1}^{I} a_{k}\right]\right]
$$

where, for every $g, u\left(\sigma g+\sum_{k=1}^{I} a_{k}, g\right) \in U$ and the emissions have to satisfy the incentive compatibility constraint

$$
h_{j}\left(a_{j}\right)+\delta\left(u_{j}\left(\sigma g+\sum_{k=1}^{I} a_{j}, g\right)-w_{j} a_{j}\right) \geq h_{j}\left(\widetilde{a}_{j}\right)+\delta\left(\underline{u}_{j}^{j}-w_{j} \widetilde{a}_{j}\right), \forall \widetilde{a}_{j}, j
$$

Since the constraint is independent of $g$ - and so is the objective function - it follows that the solution is as well.

Proof of Theorem 7: In light of our previous results, the worst equilibrium for player $i$, if the state is $g$, is a solution to the following problem:

$$
\begin{gathered}
\operatorname{Min}_{a, u(., g) \in U} h_{i}\left(a_{i}\right)+\delta\left[u_{i}\left(\sigma g+\sum_{k=1}^{I} a_{k}, g\right)-w_{i} \sum_{k=1}^{I} a_{k}\right] \\
\text { s.t. } h_{j}\left(a_{j}\right)+\delta\left(u_{j}\left(\sigma g+\sum_{k=1}^{I} a_{j}, g\right)-w_{j} a_{j}\right) \geq h_{j}\left(\widetilde{a}_{j}\right)+\delta\left(\underline{u}_{j}^{j}-w_{j} \widetilde{a}_{j}\right), \forall \widetilde{a}_{j}, j
\end{gathered}
$$

Since $u(., g) \in U$ - a state independent set - it follows that the solution to the problem is going to be state independent as well. Furthermore, by arguments that we have seen before, it is clear that if player $j$ is going to deviate (and expects the worst equilibrium for her to be the continuation regardless of how much she deviated by), then her optimal deviation is $a_{j}^{*}$. In other words, Eqs. (27) and (28) can be simplified to yield the following:

$$
\begin{gathered}
\underline{u}_{i}=\operatorname{Min}_{a, \widetilde{u} \in U} h_{i}\left(a_{i}\right)+\delta\left[\widetilde{u}_{i}-w_{i} \sum_{k=1}^{I} a_{k}\right] \\
\text { s.t. } h_{j}\left(a_{j}\right)+\delta\left(\widetilde{u}_{j}-w_{j} a_{j}\right) \geq h_{j}\left(a_{j}^{*}\right)+\delta\left(\underline{u}_{j}^{j}-w_{j} a_{j}^{*}\right), \forall j
\end{gathered}
$$

Note that it must be the case that the incentive constraint for player $i$ - whose worst equilibrium we are analyzing - is binding in the above problem. If not, we can 
perturb $a_{i}$ in such a manner - without changing $a_{j}, j \neq i$, such that $h_{i}\left(a_{i}\right)-\delta w_{i} a_{i}$ is lowered and player $i^{\prime} s$ incentive constraint continues to be satisfied. Since the other's actions do not change, their incentives are untouched and we lower the value of the minimand in Eq. (29). In light of that observation we can rewrite the problem as

$$
\begin{gathered}
\operatorname{Max}_{a, \widetilde{u} \in U} \sum_{k \neq i} a_{k} \\
\text { s.t. } h_{j}\left(a_{j}\right)+\delta\left(\widetilde{u}_{j}-w_{j} a_{j}\right) \geq h_{j}\left(a_{j}^{*}\right)+\delta\left(\underline{u}_{j}^{j}-w_{j} a_{j}^{*}\right), \forall j
\end{gathered}
$$

It immediately follows that in the solution, $\overline{\bar{a}}(i), \sum_{j \neq i} \overline{\bar{a}}_{j}(i) \geq \sum_{j \neq i} a_{j}^{*}$. We know however that there are equilibrium payoffs that are strictly higher than the BAU payoffs (for example, the third-best payoffs are strictly higher). Hence from the incentive constraints it follows that there are emission vectors for the other players that are incentive compatible and whose sum is strictly higher than the BAU emissions.

A little reflection also shows that the optimization problem of Eq. (31) must be equivalent to some $i$-less second problem. After all the higher is the equilibrium payoff $\widetilde{u}_{j}$, the easier it is to get country $j$ to emit a high amount this period (in order to punish $i$ ). From the result for second-best problems it then follows that from the second period onwards, the emission must be at some constant level $a\left(x_{-i}\right)$. The theorem is proved.

9.5. Greenhouse Trap. Proof of Theorem 8: Suppose that the initial GHG level $g_{0}$ is above the Pareto optimal steady state $\widehat{g}$. In that case, country $i$ faces a best response problem that is identical to the one it would face if the rest of the world emitted $a^{*}$ at all levels of GHG. Note that even if $i$ drops her emission to zero, the GHG level would still remain above $\widehat{g}$; after all, $\sigma g+(I-1) a^{*} \geq \sigma g+I \widehat{a}>\widehat{g}$. (Recall Condition $L-(I-1) a^{*} \geq I \widehat{a}-$ and that $\sigma \widehat{g}+I \widehat{a}=\widehat{g}$. It follows therefore that, if $g>\widehat{g}$, the best response for $i$ is to play the BAU level $a^{*}$.

Suppose instead that $g \leq \widehat{g}$. There are two possibilities for $i^{\prime} s$ emission - either the emission level is chosen to be so high that the next period's GHG stock is above $\widehat{g}$ or it is not. Consider the two cases separately.

Case 1 Emissions such that $g_{t+1}>\widehat{g}$, i.e., $a_{i}>a\left(g_{t}\right)$.

In this case, the lifetime payoffs are $h\left(a_{i}\right)-c g_{t}+\delta\left[u^{*}-w g_{t+1}\right]$, where $u^{*}$ is the BAU payoff, $u^{*}=\frac{h\left(a^{*}\right)-\delta w I a^{*}}{1-\delta}$. Substituting for the relevant expressions, the optimal deviation in this case is then determined by

$$
\operatorname{Max}_{a_{i}>a(g)} h\left(a_{i}\right)-c g+\delta\left[\frac{h\left(a^{*}\right)-\delta w I a^{*}}{1-\delta}-w\left(\sigma g+(I-1) a(g)+a_{i}\right)\right]
$$

Collecting only the terms that involve $a_{i}$ the above expression immediately simplifies to

$$
\operatorname{Max}_{a_{i}>a(g)} h\left(a_{i}\right)-\delta w a_{i}
$$


and its solution is, in fact, the BAU emission level $a^{*} \cdot{ }^{22}$ Hence, the best deviation payoff in this case reduces to

$$
u^{*}-w g+\delta w(I-1)\left(a^{*}-a(g)\right)
$$

Case 2 Emissions such that $g_{t+1} \leq \widehat{g}$, i.e., $a_{i} \leq a\left(g_{t}\right)$.

It will be more convenient to think of emission levels in this case as $a(g)-\epsilon, \epsilon>0$. Since we only need to consider one-shot deviations, and because lowering emissions unilaterally will simply delay by one period the convergence to the steady state $\widehat{g}$, it follows that the optimal deviation can be derived from the following exercise

$$
\operatorname{Max}_{\epsilon \geq 0} h(a(g)-\epsilon)+\delta\left[h\left(\widehat{a}+\frac{\sigma \epsilon}{I}\right)+c \epsilon\right]
$$

where we have used the following facts: i) an $\epsilon$ cut-back by country $i$ leaves the state of the system at $\widehat{g}-\epsilon$ in the next period and ii) that requires an additional $\frac{\sigma \epsilon}{I}$ emission by each country to bring the GHG level two periods hence up to the Pareto optimal steady state of $\widehat{g}$. It is straightforward to verify that the maximand above is concave. A sufficient condition for the maximizer to be $\epsilon=0$ (which is what we want to show) is that the derivative at that point be non-positive, i.e.,

$$
\frac{\sigma \delta}{I} h^{\prime}(\widehat{a}) \leq h^{\prime}(a(g))-\delta c
$$

By the second part of Condition $L, a^{*} \geq a(g)$, and hence by the concavity of $h$, a sufficient condition for the last inequality is

$$
\frac{\sigma \delta}{I} h^{\prime}(\widehat{a}) \leq h^{\prime}\left(a^{*}\right)-\delta c
$$

Note that $h^{\prime}(\widehat{a})=\frac{\delta I c}{1-\sigma \delta}$ and that $h^{\prime}\left(a^{*}\right)=\frac{\delta c}{1-\sigma \delta}$. After making the appropriate substitutions, it is easy to see that the two sides of the inequality are actually equal to each other (and to $\delta c \frac{\sigma \delta}{1-\sigma \delta}$ ). Hence, the optimal deviation is not to deviate.

Combining the two cases we have the following: $a(g)$ is a best response (to $a(g)$ ) provided it yields higher lifetime payoffs than $a^{*}$. Note that the lifetime payoffs to $a(g)$ are

$$
h(a(g)-w g-\delta w I a(g)+\delta \widehat{u}
$$

Elementary algebra says that the lifetime payoffs to $a(g)$ are higher if and only if

$$
h\left(a(g)-\delta w I a(g)+\delta \widehat{u} \geq h\left(a^{*}\right)-\delta w I a^{*}+\delta u^{*}+\delta w(I-1)\left(a^{*}-a(g)\right)\right.
$$

\footnotetext{
${ }^{22}$ Another way of saying what we just did is that if country $i$ is going to push the game into the BAU region, then she might as well take the unilaterally optimal action $a^{*}$ to do so.
} 
Since $h(a)-\delta w I a$ is a concave function that is maximized at $\widehat{a}$ and since $a(g) \in$ $\left[\widehat{a}, a^{*}\right]$, it follows that $h\left(a(g)-\delta w I a(g) \geq h\left(a^{*}\right)-\delta w I a^{*}\right.$. Hence a sufficient condition for the last inequality to hold is that

$$
\widehat{u} \geq u^{*}+\delta w(I-1)\left(a^{*}-a(g)\right)
$$

In turn the second term in the right-hand side is strictly smaller than $\delta w I\left(a^{*}-\widehat{a}\right)$ and so a sufficient condition for the inequality is

$$
\widehat{u} \geq u^{*}+\delta w I\left(a^{*}-\widehat{a}\right)
$$

From the definitions, it follows that the condition is equivalent to

$$
h(\widehat{a})-\delta w I \widehat{a} \geq h\left(a^{*}\right)-\delta w I a^{*}+(1-\delta) \delta w I\left(a^{*}-\widehat{a}\right)
$$

As $\delta \uparrow 1$, the inequality converges to

$$
h(\widehat{a})-w(1) I \widehat{a} \geq h\left(a^{*}\right)-w(1) I a^{*}
$$

where the present undiscounted costs $w(1)=\frac{c}{1-\sigma}$ and $\widehat{a}$ and $a^{*}$ are respectively the solutions to $\operatorname{Max} h(a)-\delta I w(1) a$ and $\operatorname{Max} h(a)-\delta w(1) a$ (and hence $\widehat{a} \neq a^{*}$ ). By definition, the last inequality is actually a strict inequality. It follows therefore that the sufficient condition for $a(g)$ to be a (strictly) better response that $a^{*}$ holds for all $\delta$ appropriately close to 1 . The theorem is proved.

Proof of Theorem 9: The proof may be found in Dutta and Radner (2004d).

\section{REFERENCES}

Abreu, Dilip, David Pearce and Ennio Stachetti, "Towards a General Theory of Discounted Repeated Games with Discounting", Econometrica 58, 1990, 1041-1065.

Barrett, S., Environment and Statecraft: The Strategy of Environmental TreatyMaking, Oxford University Press, Oxford, 2003.

Benhabib, Jess, and Roy Radner, "Joint Exploitation of a Productive Asset," Economic Theory, 2, 1992, 155-190.

Bernheim, Douglas and Debraj Ray, "Economic Growth with Intergenerational Altruism", Review of Economic Studies, 54, 1987, 227-241.

Bruce, James P., Hoesung Lee, and Erik F. Haites, eds., Climate Change 1995: Economic and Social Dimensions of Climate Change, Intergovernmental Panel on Climate Change, Cambridge University Press, Cambridge, 1996.

Cline, William R., The Economics of Global Warming, Institute for International Economics, Washington, DC, 1992. 
Committe on the Science of Climate Change, Commission on Geosciences, Environment and Resources, National Research Council, Climate Change Science: An Analysis of Some Key Questions, National Academy Press, Washington, DC, 2001.

Dasgupta, Partha, "The Population Problem: Theory and Evidence," Journal of Economic Literature, 33, 1995, 1879-102.

Dutta, Prajit K., "What Do Discounted Optima Converge to? A Theory of Discount Rate Asymptotics in Economic Models," Journal of Economic Theory, 55, 1991, 65-94.

Dutta, Prajit K., and Roy Radner, "Self-Enforcing Climate Change Treaties," Proceedings of the National Academy of Sciences, USA, 2004a, Vol. 101-14, 51745179.

Dutta, Prajit K., and Roy Radner, "Population Growth and Technological Change in a Global Warming Model", Economic Theory, forthcoming, 2004b.

Dutta, Prajit K., and Roy Radner, "Choosing Cleaner Technologies: Global Warming and Technological Change," 2004c (in preparation).

Dutta, Prajit K., and Roy Radner, "A Strategic Model of Global Warming Model: Theory and Some Numbers", mimeo, Columbia University 2004d.

Dutta, Prajit K., S. Park, and Roy Radner, "Global Warming with Capital Accumulation", in preparation, Columbia University 2004.

Dutta, P. K. and R. Sundaram, "Markovian Equilibrium in a Class of Stochastic Games: Existence Theorems for Discounted and Undiscounted Models", Economic Theory, 2, 1992, 197-214.

Dutta, P. K. and R. Sundaram, "How Different Can Strategic Models Be?", Journal of Economic Theory, 60, 1993, 42-61.

Finus, M, Game Theory and International Environmental Cooperation, Cheltenham, Edward Elgar, 2001.

Grubler, Arnulf, and Yasumasa Fujii, "Intergenerational and Spatial Equity Issues of Carbon Accounts," Energy, 16 (1991), 1397-1416.

Heal, Geoffrey M., "Interpreting Sustainability," Columbia University Business School, 1997 (unpublished).

Levhari, D. and L. Mirman, "The Great Fish War: An Example Using a Dynamic Cournot-Nash Solution", Bell Journal of Economics, 11, 1980, 322-334.

Nordhaus, William and Zili Yang, "A Regional Dynamic General Equilibrium Model of Alternative Climate-Change Strategies", American Economic Review, 86, 1996, 741-765.

Nordhaus, William and J Boyer, Warming the World: Economic Models of Global Warming, MIT Press, Cambridge, MA, 2000.

Nakicenovic, Nebojsa, "Freeing Energy from Carbon," in (Ausubel, et al, 1996, pp. 95-112). 
Park, S, "Calibrating a Model of Global Warming", Columbia University Economics Department, 2004 (unpublished).

Radner, Roy, "Dynamic Games in Organization Theory," Journal of Economic Behavior and Organization, 16, 1991, 217-260.

Rustichini, Aldo, 1992, "Second-Best Equilibria for Games of Joint Exploitation of a Productive Asset," Economic Theory, 2 1992, 191-196.

Sobel, Matthew J., "Myopic Solutions of Affine Dynamic Models," Operations Research, 38, 1990, 847-53.

Sorger, G., "Markov-Perfect Nash equilibria in a Class of Resource Games", Economic Theory, 11, 1998, 79-100.

Sundaram, R., "Perfect Equilibrium in a Class of Symmetric Dynamic Games", Journal of Economic Theory, 47, 1989, 153-177.

Thomson, David J., "Dependence of Global Temperatures on Atmospheric $\mathrm{CO}_{2}$ and Solar Irradiance," Proceedings of the National Academy of Sciences, USA, 94, 1997, 8370-77.

Victor, David G., and Eugene B. Skolnikoff, "Implementing Environmental Commitments," Options, IIASA, Spring 1997, 7-11.

Weitzman, Martin L., "Sustainability and the Welfare Significance of National Product Revisited," Discussion Paper 1737, Harvard Ins. of Econ. Res., Harvard University, 1995, (unpublished). 